\title{
Asymptotics for Bosonic Atoms
}

\author{
JAN PHILIP SOLOVEJ* \\ Department of Mathematics, University of Michigan, Ann Arbor, MI 48109, U.S.A.
}

(Received: 30 November 1989)

Abstract. It was proved by Benguria and Lieb that for an atom where the 'electrons' do not satisfy the exclusion principle, the critical electron number $N_{c}$, i.e., the maximal number of electrons the atom can bind, satisfies $\lim \inf _{\boldsymbol{Z} \rightarrow \infty} N_{c} / Z \geqslant 1+\gamma$, where $Z$ is the nuclear charge. Here $\gamma$ is a positive constant derived from the Hartree model. We complete this result by proving that the correct asymptotics for $N_{c}(Z)$ is indeed $\lim _{Z \rightarrow \infty} N_{c} / Z=1+\gamma$.

AMS subject classification (1980). 81-XX.

\section{Introduction}

In this Letter, we study the atomic Schrödinger operator

$$
H(N)=\sum_{i=1}^{N}\left(-\Delta_{i}-\frac{Z}{\left|x_{i}\right|}\right)+\sum_{1 \leqslant i<j \leqslant N} \frac{1}{\left|x_{i}-x_{j}\right|},
$$

acting on $\mathscr{H}=L^{2}\left(\mathbb{R}^{3 N}\right)$. The ground state energy of $H(N)$ is

$$
E(N)=\inf \operatorname{spec}_{\mathscr{x}} H(N) \text {. }
$$

This is the same as if we had restricted $H(N)$ to the space of functions symmetric in the $N$ space variables. $E(N)$ thus corresponds to the energy of an atom where the 'electrons' obey Bose symmetry. We define the ionization energy by

$$
I(N)=E(N-1)-E(N) \geqslant 0 .
$$

There exists $N_{c}(Z)<2 Z+1$ (see [8]) such that

$$
I\left(N_{c}(Z)\right)>0 \text { and } I(N)=0 \text { for all } N>N_{c}(Z) .
$$

$N_{c}(Z)$ is the maximal number of Bosonic 'electrons' that can be bound by the nucleus.

We will here study the asymptotics of $N_{c}$ as $Z \rightarrow \infty$. The motivation for this comes from [2], where it was proved by comparison with the Hartree model that

$$
\liminf _{Z \rightarrow \infty} \frac{N}{Z} \geqslant 1+\gamma
$$

for a constant $\gamma>0$.

* This work was done while the author was a graduate student at Princeton University supported by a Danish Rescarch Academy fellowship and U.S. National Science Foundation grant PHY-85-15288-A03. 
The Hartree model is defined by the functional

$$
L_{m}^{H}(\rho)=\int\left(m^{-1}(\nabla \sqrt{\rho(x)})^{2}-\frac{Z}{|x|} \rho(x)\right) \mathrm{d} x+D(\rho, \rho),
$$

where

$$
D(f, g)=\frac{1}{2} \int f(x)|x-y|^{-1} g(y) \mathrm{d} x \mathrm{~d} y .
$$

$L_{m}^{H}$ is defined on the set of functions $\rho \geqslant 0$ with $\sqrt{\rho} \in H^{1}\left(\mathbb{R}^{3}\right)$. The energy in this model is

$$
E_{m}^{H}(N)=\inf \left\{L_{m}^{H}(\rho) \mid \int \rho(x) \mathrm{d} x=N\right\}
$$

Then by scaling we find

$$
E_{m}^{H}(N)=-m Z^{3} e(N / Z)
$$

where $e(t)$ is a monotone nondecreasing, concave function. There exists $\rho_{m}^{H}$, such that

$$
L_{m}^{H}\left(\rho_{m}^{H}\right)=\min _{N} E_{m}^{H}(N) \equiv m \bar{E}(Z)
$$

where $\bar{E}(Z)=-Z^{3} \min e$.

It is known (see [7] where the more general Thomas-Fermi-von Weizsäcker (TFW) theory is studied) that if we write $\psi_{m}^{H}=\sqrt{\rho_{m}^{H}}$, then $\psi_{m}^{H}$ is the unique positive solution to

$$
-m^{-1} \Delta \psi_{m}^{H}-\left(\frac{Z}{|x|}-\rho_{m}^{H}|x|^{-1}\right) \psi_{m}^{H}=0
$$

and

$$
\int \rho_{m}^{H}(x) \mathrm{d} x=(1+\gamma) Z
$$

This is the same $\gamma$ as in (5). It was proved by Benguria (for a reference, see [7]) that $0<\gamma<1$. The numerical value (see [1]) is $\gamma=0.21$. If $N \geqslant(1+\gamma) Z$ then $E_{m}^{H}(N)=m \bar{E}(Z)$, i.e., $e(t)=\min e$ when $t \geqslant \gamma+1$.

Here we complete the result (5) by proving a similar upper bound, i.e.

\section{THEOREM 1.}

$$
\lim _{Z \rightarrow \infty} \frac{N_{c}(Z)}{Z}=1+\gamma
$$

In the case of fermions we have asymptotic neutrality, i.e., the above limit is 1 . This was proved in [10] and, recently, by a different method, in [4]. 


\section{Bounds on the Ground State Energy}

In this Section we improve the energy bounds from [2]. By choosing a product trial function it is easy to conclude (see [2]) that

$$
E(N) \leqslant E_{m=1}^{H}(N) \text {. }
$$

We will now prove a lower bound to $E(N)$. In fact we will prove a lower bound to the operator $H$.

For all functions $\varphi \in L^{2}\left(\mathbb{R}^{3 N}\right)$ symmetric in the $N$ space variables (but not necessarily normalized) we define the one-particle density

$$
\rho_{\varphi}(x)=N \int\left|\varphi\left(x, x_{2} \ldots, x_{N}\right)\right|^{2} \mathrm{~d} x_{2} \cdots \mathrm{d} x_{N}
$$

and the two-particle correlation

$$
\rho_{\varphi}^{(2)}(x, y)=N(N-1) \int\left|\varphi\left(x, y, \ldots, x_{N}\right)\right|^{2} \mathrm{~d} x_{3} \cdots \mathrm{d} x_{N} .
$$

To get the lower bound, we proceed as in [2]. From the simple kinetic energy inequality of Hoffmann-Ostenhof [5] (Lemma 2), we find that for any symmetric function $\varphi \in L^{2}\left(\mathbb{R}^{3 N}\right)$

$$
\begin{aligned}
\langle\varphi|H(N)| \varphi\rangle \geqslant & \int\left(\left(\nabla \sqrt{\rho_{\varphi}(x)}\right)^{2}-\frac{Z}{|x|} \rho_{\varphi}(x)\right) \mathrm{d} x+ \\
& +\sum_{1 \leqslant i<j \leqslant N}\left\langle\varphi\left|\frac{1}{\left|x_{i}-x_{j}\right|}\right| \varphi\right\rangle .
\end{aligned}
$$

We estimate the last term with the Lieb-Oxford inequality (see [6] and [9])

$$
\begin{aligned}
& \sum_{1 \leqslant i<j \leqslant N}\left\langle\varphi\left|\frac{1}{\left|x_{i}-x_{j}\right|}\right| \varphi\right\rangle \\
\geqslant & \|\varphi\|^{-2} D\left(\rho_{\varphi}, \rho_{\varphi}\right)-(1.68)\|\varphi\|^{-2 / 3} \int \rho_{\varphi}^{4 / 3} \mathrm{~d} x \\
= & \|\varphi\|^{-2} D\left(\rho_{\varphi}-\rho_{m}^{H}\|\varphi\|^{2}, \rho_{\varphi}-\rho_{m}^{H}\|\varphi\|^{2}\right)+2 D\left(\rho_{\varphi}, \rho_{m}^{H}\right)- \\
& \quad-\|\varphi\|^{2} D\left(\rho_{m}^{H}, \rho_{m}^{H}\right)-(1.68)\|\varphi\|^{-2 / 3} \int \rho_{\varphi}^{4 / 3} \mathrm{~d} x,
\end{aligned}
$$

where $\rho_{m}^{H}$ was given in (10). If we insert this above we obtain

$$
\begin{aligned}
& \langle\varphi|H(N)| \varphi\rangle \\
& \geqslant \int\left(\frac{1}{(1+\varepsilon)}\left(\nabla \sqrt{\rho_{\varphi}}\right)^{2}-\left(\frac{Z}{|x|}-\rho_{m}^{H}|x|^{-1}\right) \rho_{\varphi}(x)\right) \mathrm{d} x-\|\varphi\|^{2} D\left(\rho_{m}^{H}, \rho_{m}^{H}\right)+ \\
& \quad+\frac{\varepsilon}{1+\varepsilon} \int\left(\nabla \sqrt{\rho_{\varphi}}\right)^{2}-(1.68)\|\varphi\|^{-2 / 3} \int \rho_{\varphi}^{4 / 3} \mathrm{~d} x+ \\
& \quad+\|\varphi\|^{-2} D\left(\rho_{\varphi}-\rho_{m}^{H}\|\varphi\|^{2}, \rho_{\varphi}-\rho_{m}^{H}\|\varphi\|^{2}\right) .
\end{aligned}
$$


Using the Sobolev inequality $\|\nabla g\|_{2}^{2} \geqslant 3(\pi / 2)^{4 / 3}\|g\|_{6}^{2}$ and Hölder's inequality

$$
\int \rho_{\varphi}^{4 / 3} \leqslant\left(\int \rho_{\varphi}^{3}\right)^{1 / 6}\|\varphi\|^{5 / 3} N^{5 / 6}
$$

we easily get (see also [2])

$$
\frac{\varepsilon}{1+\varepsilon} \int\left(\nabla \sqrt{\rho_{\varphi}}\right)^{2} \mathrm{~d} x-(1.68)\|\varphi\|^{-2 / 3} \int \rho_{\varphi}^{4 / 3} \mathrm{~d} x \geqslant-a \frac{1+\varepsilon}{\varepsilon}\|\varphi\|^{2} N^{5 / 3},
$$

where $a=0.13$. We now choose $m=1+\varepsilon$. Since $\psi_{m}^{H}$ satisfies the Hartree equation (11) we see that $m \bar{E}(Z)=-D\left(\rho_{m}^{H}, \rho_{m}^{H}\right)$ and since $\psi_{m}^{H} \geqslant 0, \psi_{m}^{H}$ is the ground state of

$$
-m^{-1} \Delta-\left(\frac{Z}{|x|}-\rho_{m}^{H} *|x|^{-1}\right)
$$

This is thus a positive operator. We can therefore conclude that

$$
\begin{aligned}
\langle\varphi|H(N)| \varphi\rangle \geqslant & \left((1+\varepsilon) \tilde{E}(Z)-a \frac{1+\varepsilon}{\varepsilon} N^{5 / 3}\right)\|\varphi\|^{2}+ \\
& +\|\varphi\|^{-2} D\left(\rho_{\varphi}-\rho_{m}^{H}\|\varphi\|^{2}, \rho_{\varphi}-\rho_{m}^{H}\|\varphi\|^{2}\right) .
\end{aligned}
$$

Using $N<c Z$ and choosing $\varepsilon=c Z^{-2 / 3}$ we have proved

LEMMA 2. For all symmetric $\varphi \in L^{2}\left(\mathbb{R}^{3 N}\right)$ we have

$$
\langle\varphi|H(N)| \varphi\rangle \geqslant\left(\bar{E}(Z)-c Z^{7 / 3}\right)\|\varphi\|^{2}+\|\varphi\|^{-2} D\left(\rho_{\varphi}-\rho_{m}^{H}\|\varphi\|^{2}, \rho_{\varphi}-\rho_{m}^{H}\|\varphi\|^{2}\right),
$$

where $m=1+c Z^{-2 / 3}$.

\section{Structure of Ground State}

In this section we will prove Theorem 1. The main technical step is (in the fermionic case a similar result is given in [11])

LEMMA 3. Let $\chi, \theta \in C^{1}\left(\mathbb{R}^{3}\right)$ be nonnegative functions with $\chi$ compactly supported. If $N \geqslant(1+\gamma) Z$ and $H(N)$ has a ground state $\psi$ we then have the following estimate involving the ground state density $\rho=\rho_{\psi}$ and two point correlation $\rho^{(2)}=\rho_{\psi}^{(2)}$

$$
\begin{aligned}
& \left(\int\left[\rho^{(2)}(x, y)-\rho_{m}^{H}(y) \rho(x)\right] \theta(x) \chi(y) \mathrm{d} x \mathrm{~d} y\right)^{2} \\
& \quad \leqslant C\|\nabla \chi\|^{2}\left[Z^{7 / 3}\left(\int \rho(x) \theta(x) \mathrm{d} x\right)^{2}+Z\|\nabla \sqrt{\theta}\|_{\infty}^{2} \int \rho(x) \theta(x) \mathrm{d} x\right],
\end{aligned}
$$

where $m=1+c Z^{-2 / 3}$.

Proof. Since the ground state $\psi$ of $H(N)$ is symmetric we can write

$$
\begin{aligned}
E(N) \int \rho \theta \mathrm{d} x & =\sum_{i=1}^{N}\left\langle\psi\left|\theta\left(x_{i}\right) H(N)\right| \psi\right\rangle \\
& =\sum_{i=1}^{N}\left\langle\psi\left|\theta\left(x_{t}\right)^{1 / 2} H(N) \theta\left(x_{i}\right)^{1 / 2}-\left(\nabla \sqrt{\theta\left(x_{i}\right)}\right)^{2}\right| \psi\right\rangle,
\end{aligned}
$$


where in the last equality we have used a simple commutation identity also used in the IMS formula (see [3]). If we let $H^{(i)}(N-1)$ denote the operator with the $i$ th variable removed and use that the lowest eigenvalue of $-\Delta-(Z /|x|)$ is $-\frac{1}{4} Z^{2}$ we get

$$
\begin{gathered}
E(N) \int \rho \theta \mathrm{d} x \geqslant \\
\sum_{i=1}^{N}\left\langle\psi\left|\theta\left(x_{i}\right)^{1 / 2} H^{(i)}(N-1) \theta\left(x_{i}\right)^{1 / 2}\right| \psi\right\rangle- \\
-N\|\sqrt{\theta}\|_{\infty}^{2}-\frac{1}{4} Z^{2} \int \rho \theta \mathrm{d} x
\end{gathered}
$$

where in the $i$ th term we have neglected the repulsion between the $i$ th electron and all the rest. If we now use Lemma 2 for $H^{(i)}(N-1)$ with $\varphi_{i}=\theta\left(x_{i}\right)^{1 / 2} \psi$ (here $x$, is a parameter) we get

$$
\begin{aligned}
E(N) \int \rho \theta \mathrm{d} x \geqslant & \left(\bar{E}(Z)-c Z^{7 / 3}\right) \int \rho \theta \mathrm{d} x-N\|\nabla \sqrt{\theta}\|_{\infty}^{2}+ \\
& +\sum_{i=1}^{N} \int\left\|\varphi_{i}\right\|^{-2} D\left(\rho^{(i)}-\rho_{m}^{H}\left\|\varphi_{i}\right\|^{2}, \rho^{(i)}-\rho_{m}^{H}\left\|\varphi_{i}\right\|^{2}\right) \mathrm{d} x_{i},
\end{aligned}
$$

here

$$
\rho^{(i)}(x)=\rho_{\varphi_{i}}(x)=N^{-1} \theta\left(x_{i}\right) \rho^{(2)}\left(x, x_{i}\right)
$$

and

$$
\left\|\varphi_{i}\right\|^{2}=(N-1)^{-1} \int \rho^{(i)}(x) \mathrm{d} x=N^{-1} \theta\left(x_{i}\right) \rho\left(x_{i}\right) .
$$

We can therefore write

$$
\begin{aligned}
& \int\left[\rho^{(2)}(x, y)-\rho_{m}^{H}(y) \rho(x)\right] \theta(x) \chi(y) \mathrm{d} x \mathrm{~d} y \\
& =\sum_{i=1}^{N} \int\left(\rho^{(i)}(y)-\left\|\varphi_{i}\right\|^{2} \rho_{m}^{H}(y)\right) \chi(y) \mathrm{d} x_{i} \mathrm{~d} y .
\end{aligned}
$$

We use the Fourier transform (denoted ${ }^{\wedge}$ ) to estimate the last expression. This idea comes from [4]. It is here that we need some decay of $\chi$.

$$
\begin{aligned}
& \left(\int\left(\rho^{(i)}(y)-\left\|\varphi_{i}\right\|^{2} \rho_{m}^{H}(y)\right) \chi(y) \mathrm{d} y\right)^{2} \\
& \quad=\left(\int\left(\rho^{(i)}-\left\|\varphi_{i}\right\|^{2} \rho_{m}^{H}\right)^{\wedge}(p) \hat{\chi}(p) \mathrm{d} p\right)^{2} \\
& \quad \leqslant\left.\int|\hat{\chi}|^{2}|p|^{2} \mathrm{~d} p \int\left|\left(p^{(i)}-\left\|\varphi_{i}\right\|^{2} \rho_{m}^{H}\right)^{\wedge}(p)\right|\right|^{2}|p|^{-2} \mathrm{~d} p \\
& \quad \text { const }\|\nabla \chi\|^{2} D\left(\rho^{(i)}-\rho_{m}^{H}\left\|\varphi_{i}\right\|^{2}, \rho^{(i)}-\rho_{m}^{H}\left\|\varphi_{i}\right\|^{2}\right)
\end{aligned}
$$


and using Cauchy-Schwarz inequality

$$
\begin{aligned}
& \left(\int\left(\rho^{(i)}(y)-\left\|\varphi_{i}\right\|^{2} \rho_{m}^{H}(y)\right) \chi(y) \mathrm{d} y \mathrm{~d} x_{i}\right)^{2} \\
& \quad \leqslant \int\left(\int\left(\rho^{(i)}(y)-\left\|\varphi_{i}\right\|^{2} \rho_{m}^{H}(y)\right) \chi(y) \mathrm{d} y\right)^{2}\left\|\varphi_{i}\right\|^{-2} \mathrm{~d} x_{i} \int\left\|\varphi_{i}\right\|^{2} \mathrm{~d} x_{i} \\
& \quad \leqslant C\|\nabla \chi\|^{2} \int D\left(\rho^{(i)}-\rho_{m}^{H}\left\|\varphi_{i}\right\|^{2}, \rho^{(i)}-\rho_{m}^{H}\left\|\varphi_{i}\right\|^{2}\right)\left\|\varphi_{i}\right\|^{-2} \mathrm{~d} x_{i} N^{-1} \int \rho \theta \mathrm{d} x .
\end{aligned}
$$

Since $N \geqslant(1+\gamma) Z$ we know that $E_{m=1}^{H}(N)=\bar{E}$ and hence from (14), $E(N) \leqslant \bar{E}(Z)$. We can estimate the above expression from (16) where the terms in the sum are independent of $i$. Inserting this into (17) and noticing again that the terms in the sum of (17) are all the same, we arrive at the final result (recall that $N<2 Z+1)$.

EXAMPLE. If we choose $\theta=1$ in the Lemma we find with $m=1+c Z^{-2 / 3}$

$$
\left((N-1) \int \rho(y) \chi(x) \mathrm{d} y-N \int \rho_{m}^{H}(y) \chi(y) \mathrm{d} y\right)^{2} \leqslant C N^{2} Z^{7 / 3}\|\nabla \chi\|^{2}
$$

or

$$
\left(\int \rho(y) \chi(x) \mathrm{d} y-\int \rho_{m}^{H}(y) \chi(y) \mathrm{d} y\right)^{2} \leqslant C Z^{7 / 3}\|\nabla \chi\|^{2} .
$$

We can now give the

Proof of Theorem 1. Because of the lower bound in [2], we only have to prove a similar upper bound. We can of course assume that $N_{c}>(1+\gamma) Z$. We are now concentrating on the case $N=N_{c}$. Since $I\left(N_{c}\right)>0$ it follows from the HVZ Theorem (see [3]) that $H\left(N_{c}\right)$ has a ground state.

First choose localizing functions $\chi_{R}, \theta_{R}$ as in the Lemma, but depending on a scale $R . \chi_{R}$ should be supported in a ball of radius $R$ around the origin and be equal to 1 in a ball of radius $\frac{1}{2} R$. Furthermore, we want $\chi_{R}+\theta_{R}=1$. Then

$$
\left\|\nabla \chi_{R}\right\|^{2} \leqslant c R \text { and }\left\|\nabla \sqrt{\theta_{R}}\right\|_{\infty} \leqslant c R^{-1} .
$$

We will use (18) to give a bound on $\int \rho(x) \chi_{R}(x) \mathrm{d} x$. From the scaling of the Hartree model we find that

$$
\rho_{m}^{H}(x)=m^{3} Z^{4} \rho^{H}(m Z x),
$$

where $\rho^{H}$ is the Hartree density corresponding to $Z=1$ and $m=1$. It follows from the results in [7] on the TFW theory, that $\rho^{H}$ is exponentially decaying at infinity. Now choose $R=Z^{-7 / 9}$. Then it follows immediately from (18) that (recall that $\left.m=1+c Z^{-2 / 3}\right)$

$$
\left|\int \rho(x) \chi_{R}(x) \mathrm{d} x-(1+\gamma) Z\right| \leqslant C Z^{7 / 9} \text {. }
$$


To estimate $\int \rho(x) \theta_{R}(x) \mathrm{d} x$ we proceed as in the beginning of the proof of Lemma 3 and use the IMS formula to prove

$$
\begin{aligned}
& E(N) \int \rho(x) \theta_{R}(x)|x| \mathrm{d} x \\
& \geqslant E(N-1) \int \rho(x) \theta_{R}(x)|x| \mathrm{d} x-Z \int \rho(x) \theta_{R}(x) \mathrm{d} x+ \\
& \quad+\int \rho^{(2)}(x, y) \theta_{R}(x)|x||x-y|^{-1} \mathrm{~d} x \mathrm{~d} y-N\left\|\nabla \sqrt{|x| \theta_{R}}\right\|_{\infty}^{2},
\end{aligned}
$$

here we have kept the repulsion between the $i$ th electron and the others but neglected the $-\Delta_{i}$ term. We choose $0<\lambda<\gamma / 2$ and restrict the $y$-integral above to $|y| \leqslant \lambda R$, i.e., $y \in \operatorname{supp} \chi_{\lambda R}$. If $x \in \operatorname{supp} \theta_{R}$ and $y \in \operatorname{supp} \chi_{\lambda R}$ then $|x-y| \leqslant$ $|x|+\lambda R \leqslant(1+2 \lambda)|x|$. Hence, from (4)

$$
0 \geqslant-Z \int \rho(x) \theta_{R}(x) \mathrm{d} x+\frac{1}{1+2 \lambda} \int \rho^{(2)}(x, y) \theta_{R}(x) \chi_{\lambda R}(y) \mathrm{d} x \mathrm{~d} y-c N R^{-1} .
$$

We then apply Lemma 3 to estimate the second term, again using the results on $\rho^{H}$ mentioned above

$$
\begin{aligned}
0 \geqslant & \left(-Z+\frac{1+\gamma}{1+2 \lambda} Z\right) \int \rho(x) \theta_{R}(x) \mathrm{d} x-c N R^{-1}- \\
& -c R^{1 / 2} Z^{7 / 6} \int \rho(x) \theta_{R}(x) \mathrm{d} x-c R^{-1 / 2} Z^{1 / 2}\left(\int \rho(x) \theta_{R}(x) \mathrm{d} x\right)^{1 / 2} .
\end{aligned}
$$

Since $2 \lambda<\gamma$ and $\int \rho \theta_{R} \leqslant$ const $Z$ we find

$$
c\left(Z-Z^{7 / 9}\right) \int \rho(x) \theta_{R}(x) \mathrm{d} x \leqslant c Z^{16 / 9} .
$$

This then implies the final estimate

$$
N_{c}=\int \rho(x) \mathrm{d} x=\int \rho(x) \chi_{R}(x) \mathrm{d} x+\int \rho(x) \theta_{R}(x) \mathrm{d} x \leqslant(1+\gamma) Z+c Z^{7 / 9}
$$

from which Theorem 1 follows.

\section{References}

1. Baumgartner, B., On the Thomas-Fermi-von Weizsäcker and Hartree energies as functions of the degree of ionization, J. Phys. A 17, 1593-1602 (1984).

2. Benguria, R. and Lieb, E. H., Proof of stability of highly negative ions in the absence of the Pauli principle, Phys. Rev. Lett. 50, $1771-1774$ (1983).

3. Cycon, H. L., Froese, R. G., Kirsch, W., and Simon, B., Schrödinger Operators, Springer-Verlag, Berlin, Heidelberg, 1987.

4. Fefferman, C. L. and Seco, L. A., Asymptotic neutrality of large ions, Comm. Math. Phys., to appcar. 
5. Hoffmann-Ostenhof, M. and Hoffmann-Ostenhof, T., 'Schrödinger inequalities' and asymptotic behavior of the electron density of atoms and molecules, Phys. Rev. A 16, 1782-1785 (1977).

6. Lieb, E. H., A lower hound for Coulomb energies, Phys. Lett. 70A, 444-446 (1979).

7. Lieb, E. H., Thomas-Fermi and related theories of atoms and molecules, Rev. Mod. Phys. 53, 603-641 (1981).

8. Lieb, E. H., Bound on the maximum negative ionization of atoms and molecules, Phys. Rev. A 29, 3018-3028 (1984).

9. Lieb, E. H. and Oxford, S., An improved lower bound on the indirect Coulomb energy, Int. J. Quantum Chem. 19, 427-439 (1981).

10. Lieb, E. H., Sigal, I. M., Simon, B., and Thirring, W., Asymptotic neutrality of large-Z ions, Comm. Math. Phys. 116, 635-644 (1988).

11. Seco, L. A., Sigal, I. M., and Solovej, J. P., Bound on the ionization energy of large atoms, Comm. Math. Phys., to appear. 